\title{
Point and Density Forecasts of Oil Returns: The Role of Geopolitical Risks ${ }^{\#}$
}

\author{
Vasilios Plakandaras ${ }^{+*}$, Rangan Gupta ${ }^{* *}$ and Wing-Keung Wong ${ }^{* * *}$ \\ * Department of Economics, Democritus University of Thrace, Greece \\ Email: vplakand@econ.duth.gr \\ ***Department of Economics, University of Pretoria, South Africa. \\ Email: rangan.gupta@up.ac.za
}

${ }^{* * *}$ Department of Finance, , Fintech Center, and Big Data Research Center, Asia University; Department of Medical Research, China Medical University Hospital, Taiwan; Department of

Economics and Finance, Hang Seng Management College, Hong Kong;

Department of Economics, Lingnan University, Hong Kong.

Email: wong@asia.edu.tw

\section{Highlights}

- We examine the dynamic relationship between oil prices and geopolitical risk.

- We use linear and nonlinear probabilistic models.

- Oil prices are affected by war threats and acts only in the short run.

- From density forecasts oil prices can be predicted during periods of low risk.

\begin{abstract}
We examine the dynamic relationship between oil prices and news-based indices of global geopolitical risks (GPRs), as well as a composite measure of the same for emerging economies, which we develop using Dynamic Model Averaging (DMA). In doing so, we train a number of linear and nonlinear probabilistic models to capture the ability of GPRs in forecasting oil returns. Our empirical findings show that global GPRs associated with wars is the most accurate in forecasting oil returns in the short-run, while composite GPRs emanating from the emerging markets, forecasts oil returns relatively better at medium- to longer-horizons. However, differences across the linear and nonlinear models incorporating information of GPRs are not necessarily markedly different. Given an observed negative relationship between GPRs and oil returns, density forecasts show that increases in GPRs from their initial lower levels, which would imply higher conditional oil returns initially, can predict the resulting increases in oil returns thereafter more accurately compared to the lower end of the conditional distribution, which in turn, corresponds to higher initial levels of GPRs.
\end{abstract}

Keywords: Bayesian VAR, Geopolitical Risks, Oil Prices, Dynamic Model Averaging

JEL Codes: C22, C32, Q41, Q47

\footnotetext{
\# We would like to thank two anonymous referee and the editor of the journal for many helpful comments. Naturally, all remaining errors are solely ours.

${ }^{+}$Corresponding author.
} 


\section{Introduction}

Oil price movements are known to predict recessions (Hamilton, 1983, 2008, 2009, 2013), as well as inflation (Stock and Watson, 2003). In other words, oil prices act as a leading indicator for the macroeconomy. Moreover, oil market movements are widely known to spillover to other commodity markets, as well as equity, bond, and currency markets (see, Gupta and Yoon (2018) for a detailed discussion of this literature), i.e., oil prices contain useful information about the future path of movement of other commodities and financial markets. Additionally, oil is indispensable for industrial, transportation, and agricultural sectors, whether used as feedstock in production or as a surface fuel in consumption (Mensi, et al., 2014).

Hence, oil prices can affect the future profitability of industries in the economy. Given that the impact of oil prices span not only the aggregate economy, but also at firm- and sectoral-levels, accurate prediction of oil price movements is of importance to academics, investors and policymakers alike. Understandably, there exists a large literature (see for example, Baumeister (2014), Degiannakis and Filis (2017), and Gupta and Wohar (2017) for detailed reviews) aiming to predict oil price movements using various types of econometric methodologies (univariate and multivariate; linear and nonlinear), and predictors (macroeconomic, financial, behavioural, institutional).

In this regard, more recent studies by Bloomberg et al., (2009), Fattouh (2011), Antonakakis et al., (2017a, b), Caldara and Iacoviello (2018), Cunado et al., (2018), Demirer et al., (forthcoming), have related oil price movements with geopolitical risks (GPRs). These studies point out that, since GPRs affect the economic conditions of both developed and emerging markets, and oil prices are functions of the state of the economy, it is expected intuitively, that oil market movements are likely to be affected by risks associated with geopolitical events through the oil-demand channel. In addition, with GPRs also affecting financial markets (Balcilar et al., 2018), and with oil and financial markets connected closely, such risks can also affect the oil prices indirectly through asset markets.

Against this backdrop, given the importance of the knowledge about the future trends in oil market at the macroeconomic and sectoral-levels, we aim to add to this literature by analysing the ability of global and emerging market GPRs in forecasting 
West Texas Intermediate (WTI) oil return. For our purpose, we investigate the monthly period of January 1985 to June 2017, and generate both point and density forecasts from various linear and nonlinear models. While point forecasts are important, density forecasts provide a measure of uncertainty surrounding the point forecasts (due to model specification) as well as the uncertainty in measuring the data. Density forecasts are also of immense importance in designing risk-management strategies.

All the above-mentioned studies relating GPR with movements in the oil markets (to be discussed in detail in the next section) are however, based on in-sample analysis. Campbell (2008) points out that, the ultimate test of any predictive model (in terms of the econometric methodologies and the predictors used) is in its out-of-sample performance. Since existence of in-sample predictability does not necessarily ensure out-of-sample forecasting gains (Rapach and Zhou, 2013), in this paper, we make the first attempt in forecasting oil market movements based on GPRs. As indicated above, forecasting oil price movements out-of-sample is important for providing information of the future path of growth, inflation, other commodity and asset prices, as well as firm-level profitability.

An important issue to highlight at this stage is the realization that measuring geopolitical risks, which has traditionally been associated with terror attacks only, and hence modelled via a dummy, is much broader and hence, not straight-forward to capture, and incorporate into time-series models involving continuous data. However, Caladara and Iacoviello (2018), and Caldara et al., (2018), construct monthly indices of GPRs by counting the occurrence of words related to geopolitical tensions in leading international newspapers, and circumvent the above-mentioned issues. The authors search for articles containing references to words associated with: explicit mentions of geopolitical risk, as well as mentions of military-related tensions involving large regions of the world and a U.S. involvement; nuclear tensions; war threats and terrorist threats; actual adverse geopolitical events (as opposed to just risks) which can be reasonably expected to lead to increases in geopolitical uncertainty, such as terrorist acts or the beginning of a war. Given this, in our forecasting exercise, we use the various GPRs indexes developed by these authors.

In this paper, we find that global GPRs associated with wars is the most accurate 
indicator in point-forecasting of oil returns in the short-run, while composite GPRs emanating from the emerging markets, point-forecast oil returns relatively better at medium to longer horizons. As far as density forecasts are concerned, we observe that increases in GPRs can predict resulting increases in oil returns more accurately compared to the lower end of the conditional distribution expressing drops in GPRs, suggesting an asymmetry that can be attributed to an overreacting behavior of the oil market in the presence of increased geopolitical risk.

The remainder of the paper is organized as follows: Section 2 discusses the literature on GPRs and oil market, while Section 3 presents the data and methodology. Section 4 discusses the results, with Section 5 concluding the paper.

\section{Literature on Geopolitical Risks and Oil Market Movements}

Given the widespread role of the oil market, the literature on what drives oil prices is massive and beyond the scope of this paper. However, the reader can refer to Baumeister (2014), Degiannakis and Filis (2017), and Gupta and Wohar (2017) for detailed reviews. While traditionally, oil market movements have been related with macroeconomic and financial factors, more recently studies have indicated at the role of risks associated with geopolitics in driving oil prices. In this regard, a first group of studies analyses how geopolitical risks directly affects oil returns and volatility, while in the second set of papers, the focus is on how GPRs affect the co-movements of oil and stock markets.

As far as the first-line of research is concerned, Bloomberg et al., (2009) use a panel dataset to investigate the relationship between oil profitability and conflict, compiled of conflict data from the top 20 oil producing and exporting countries in the world. This study shows that in the later part of the sample, 1974-2005, as cartel behavior of OPEC member countries has diminished and as conflict has become more regular and thus the information surrounding it noisier, oil stock prices do not increase in response to conflict. However, in earlier capacity constrained eras, oil stocks are found to increase in response to conflict, as much as by 10 percentage points. Along similar lines, Fattouh (2011) related the Libyan disruption with oil price movements that followed thereafter. The study suggests that fears of similar events engulfing other key oil exporters have caused market players to update their beliefs about the 
probability of disruptions from the region. This process of updating beliefs plus the fact that there has been an actual loss of output induced changes in price levels. In addition, sharp price rises and increased volatility have also raised doubts about the effectiveness of the market mechanisms and the role of speculators in the oil price formation process.

More recently, Caldara and Iacoviello (2018) indicates that GPRs tend to reduce oil prices based on a structural vector autoregressive (SVAR) model of the global economy. This finding is confirmed by Cunado et al., (2018), who uses a timevarying SVAR model of the oil economy to show that GPRs tend to negatively impact oil demand and hence, reduce oil prices. These findings challenged the idea that higher geopolitical risk drives oil prices up persistently (as in Bloomberg et al., 2009) - a view that might reflect a selective memory whereby one tends to confound all geopolitical tensions with oil supply shocks driven by geopolitical tensions in the Middle East. Finally, Demirer et al., (forthcoming), uses a $k$-th order causality-inquantiles test to show that GPRs not only predicts oil returns, but also oil market volatility, with the effect being stronger at the lower end of the conditional distribution.

In terms of the second line of research, Antonakakis (2017a) studied the connectedness between oil shocks and stock market returns and volatility, and suggested that connectedness varies across different time periods, with this timevarying character being aligned with certain developments that take place in the global economy. In particular, aggregate demand shocks appear to act as the main transmitters of shocks to stock markets during periods characterised by economicdriven events, while supply-side and oil-specific demand shocks during periods of geopolitical unrest - a finding in line with Cunado et al., (2018). At the same time, Antonakakis et al., (2017b), using monthly stock and oil data that spans over a century, analyze whether the time-varying stock-oil covariance, their returns and their variances are affected by GPRs. The results reveal that geopolitical risk triggers a negative effect, mainly on oil returns and volatility, and to a smaller degree on the covariance between the two markets.

As can be observed, all the above-mentioned studies relating GPR with movements in the oil markets are based on in-sample analysis. Though such structural analysis is of 
tremendous importance, but, it has been pointed out that the ultimate test of any predictive model is in its out-of-sample performance. Since existence of in-sample predictability does not necessarily ensure out-of-sample forecasting gains, in this paper, we make the first attempt in forecasting oil market movements based on GPRs. This in turn, is important since the future trends in the oil market determines the future path for the macroeconomy, other commodity and financial markets, and also profitability at the firm-level. This serves as the main distinguishing feature of our analysis, whereby we aim to produce point and density forecasts of the oil market based on information of GPRs.

\section{Data and Methodology}

\subsection{The Data}

We compile monthly WTI crude oil prices in U.S. dollars from the Global Financial Database that span the period January 1985 to June 2017 and compute their logarithmic returns. We choose to work with returns to avoid non-stationarity issues, as required by our forecasting models. The start and end dates of our analysis are purely driven by the availability of the data. The political risk is expressed by the Geopolitical Risk (GPR) indices ${ }^{1}$ of Caldara and Iacoviello (2018) and Caldara et al. (2018) in monthly frequency for the global and 18 emerging economies for the aforementioned time period. The indices are derived by the frequency of the usage of a selection of words in eight American, two British, and one Canadian newspapers.

While there exists only one index for each of the sample of the 18 emerging countries, the global index comes in five different versions: a) a simple version that identifies the frequency of a selected "bag" of words in the spirit of Baker et al. (2016); b) a narrow version that pre-processes the number of articles in order to increase their relevance to political risk; c) a broad version that uses bigrams in the annotation of the articles; d) an index that includes strictly war acts, and finally; e) an index that includes only terrorist acts. As Caldara and Iacoviello (2018) report, their indices exhibit a correlation of 0.69 with the economic policy uncertainty index of Baker et al. (2016) that is high but not an extreme value, while they better capture political events. The descriptive statistics for all variables are reported in Table 1. Most indices

\footnotetext{
${ }^{1}$ The complete dataset can be found at: https://www2.bc.edu/matteo-iacoviello/gpr.htm.
} 
exhibit positive skewness around 1, denoting a concentration of the mass of the distribution to the left of the distribution, while the significant values of kurtosis above 3 indicate leptokurtic distributions with more extreme values than the normal distribution. These characteristics are taken into consideration in the estimation of the forecasting models.

\begin{tabular}{|c|c|c|c|c|c|}
\hline \multicolumn{6}{|c|}{ Table 1: Descriptive Statistics } \\
\hline & Mean & $\begin{array}{l}\text { Standard } \\
\text { Deviation }\end{array}$ & Kurtosis & Skewness & $\begin{array}{c}\text { Jarque-Bera } \\
\text { normality } \\
\text { test }\end{array}$ \\
\hline \multicolumn{6}{|l|}{ Panel A: Oil prices } \\
\hline Oil returns & 0.001 & 0.09 & 5.61 & -0.41 & $121.02 *$ \\
\hline \multicolumn{6}{|c|}{ Panel B: Geopolitical Risk Indices of Caldara and Iacoviello (2018) } \\
\hline Global simple & 80.67 & 59.65 & 22.25 & 3.68 & $6901.59 *$ \\
\hline Global Terrorist acts & 81.06 & 63.92 & 25.25 & 3.93 & $9050.84^{*}$ \\
\hline Global War acts & 79.22 & 67.48 & 25.28 & 4.03 & $9121.09 *$ \\
\hline Global Broad & 91.65 & 38.79 & 17.73 & 3.16 & $4174.79 *$ \\
\hline Global Narrow & 81.28 & 62.44 & 24.15 & 3.83 & $8222.57 *$ \\
\hline Argentina & 117.96 & 48.03 & 6.53 & 1.43 & $335.48^{*}$ \\
\hline Brazil & 104.31 & 29.79 & 4.76 & 1.02 & $118.51 *$ \\
\hline China & 103.83 & 26.31 & 7.15 & 1.69 & $466.06^{*}$ \\
\hline Colombia & 84.94 & 27.18 & 3.41 & 0.61 & $26.82^{*}$ \\
\hline India & 97.38 & 30.20 & 8.35 & 1.97 & $717.39 *$ \\
\hline Indonesia & 79.96 & 32.19 & 8.00 & 1.56 & $564.60 *$ \\
\hline Israel & 86.85 & 22.76 & 4.66 & 1.18 & $135.43 *$ \\
\hline Korea & 109.63 & 38.67 & 6.20 & 1.40 & $293.78 *$ \\
\hline Malaysia & 95.81 & 36.53 & 8.17 & 1.66 & $613.78 *$ \\
\hline Mexico & 96.71 & 21.91 & 5.20 & 1.18 & $169.55^{*}$ \\
\hline Philippines & 103.74 & 36.99 & 3.12 & 0.64 & $26.54 *$ \\
\hline Russia & 109.49 & 30.31 & 4.96 & 1.26 & $166.43 *$ \\
\hline Saudi Arabia & 94.80 & 33.98 & 3.50 & 0.76 & $41.52 *$ \\
\hline South Africa & 123.35 & 52.68 & 3.62 & 1.03 & $75.51^{*}$ \\
\hline Thailand & 100.82 & 40.99 & 8.00 & 1.83 & $623.44 *$ \\
\hline Turkey & 112.94 & 43.98 & 5.42 & 1.32 & $208.04 *$ \\
\hline Ukraine & 124.39 & 63.77 & 3.94 & 1.11 & $94.12^{*}$ \\
\hline Venezuela & 83.80 & 37.62 & 4.50 & 0.95 & $95.62 *$ \\
\hline \multicolumn{6}{|c|}{ Panel C: Composite Index } \\
\hline DMS returns & -0.05 & 0.14 & 4.56 & 0.74 & $75.46^{*}$ \\
\hline DMA returns & -0.06 & 0.52 & 7.93 & 1.80 & $604.98 *$ \\
\hline DMS volatility & -0.05 & 0.13 & 4.23 & 0.59 & $47.12^{*}$ \\
\hline DMA volatility & -0.06 & 0.52 & 7.91 & 1.80 & $600.77 *$ \\
\hline
\end{tabular}

Note: Rejection of the null hypothesis about normality at the 5\% level of significance is marked with an asterisk. DMS stands for Dynamic Model Selection, while DMA stands for Dynamic Model Averaging. The sample spans the period from January 1985- June 2017.

Unlike the global indices that we study their ability to forecast oil returns, we use the 
GPR indices of the 18 emerging economies to construct composite GPR indices by using the Dynamic Model Selection (DMS) and the Dynamic Model Averaging (DMA) methodologies of Koop and Korobilis (2012). Our selection in constructing a composite index is motivated by the small political and economic significance of each emerging economy to the global oil market. A composite index exploits their cumulative political position, thus creating a larger "critical" political mass. The selection of the DMS and the DMA methodologies over other dimension reduction techniques (such as dynamic factor analysis) seems ideally suited for the problem of forecasting oil prices based on GPR, since they allow for the forecasting model to change over time while, at the same time, allowing for coefficients in each model to evolve over time. Moreover, DMA and DMS lifts the problem of having to select the number of factors that we will use in our estimations, as in Principal Component Analysis of Dynamic factor Analysis.

The descriptive statistics of the composite indices are reported in Panel C of Table 1. The basic idea behind using the DMS is to select the index in each step with the lowest forecasting error in one-period ahead forecasting under a Bayesian framework. Thereafter, working backwards based on the posterior probabilities, a factor variable is constructed. In contrast, the DMA uses all indices contemporaneously by assuming that each index has a different contribution to forecasting. Thus, it constructs a timevarying weighted composite index that exploits asymmetrically each index in the onestep-ahead forecasting under the Bayesian framework.

\subsection{Methodologies}

\subsubsection{Forecasting models}

To take care of the geopolitical risk that varies both in time and in severity, we employ TVP-VAR models with different Bayesian methods, in which time-varying VAR coefficients are allowed with stochastic volatility on the innovations (Primiceri, $2005^{2}$ ), to capture possible heteroscedasticity of the shocks and potential nonlinearities in the dynamic relationships of the variables. The selection of the TVP-

\footnotetext{
${ }^{2}$ Primiceri (2005) initially proposed his model in order to study monetary phenomena and not in forecasting as we do in this paper. Later, the same model is used in detecting causal relationships between variables (Nasir et al., 2018). There is no reported use in the literature of the same model in forecasting economic variables and especially based on geopolitical risk.
} 
VAR over other forecasting methodologies is motivated by the fact that despite the vast use of VAR models in the literature there exist limited use in forecasting oil prices based on GPR. So, it is interesting to observe the forecasting performance of VAR models in this application. Our approach is also coupled with the threshold notion in segregating low and high geopolitical tensions to different models and we also relax the homoskedasticity assumption to address real data. Moreover, we modify the approach used by Chiu et al. (2017) to assume that the innovations follow the Student's $t$-distribution to provide an effective treatment of outliers and extreme events on increased volatility. We denote this model TVP-tVAR. In addition, to account for the extant literature of Markov Switching (MS) class of models of oil prices (Balcilar et al., 2015), we also consider an MS-VAR model with time-varying transition probabilities and its counterparts Threshold VAR (TVP-TVAR) and a version based on the Student's $t$-distribution coded TVP-tTVAR. Of course, this list of models is by no way exhausting, but we attempt to address the forecasting problem based on the typical models used in the econometric literature.

We start with a simple VAR model:

$$
Y_{t}=c+\theta_{t}(L) x_{t}+\varepsilon_{t},
$$

in which $x_{t}$ is a $n \times 1$ vector of endogenous variables (namely, oil returns/volatility and the GPR index that will be examined) at time $t$, each $\theta_{j t}$ in $\theta_{t}(L)=I-\theta_{1 t} L-$ $\cdots-\theta_{1 t} L^{1}$ is a matrix of time-varying coefficients, and $\varepsilon_{t}$ is a vector of zero mean VAR errors with a time-varying covariance matrix $R_{t}$. The coefficient in (1) evolves according to:

$$
\theta_{t}=\theta_{t-1}+u_{t}
$$

with $\theta_{t}$ denoting the vector that stacks all parameters in $\theta_{t}(L)$ and $u_{t}$ being a Gaussian white noise process with zero mean and constant covariance matrix $Q$, independent of $\varepsilon_{t}$ at all leads and lags. We model the time variations of innovations $\mathrm{R}_{t}=\mathrm{E}\left(e_{t} e_{t}^{\prime}\right)=F_{t} D_{t} F_{t}^{\prime}$, where $F_{t}$ is a lower diagonal matrix with ones in the main diagonal and $D_{t}$ a diagonal matrix. In order to provide flexibility to our model, we drop the typical homoscedasticity assumption and include stochastic volatility on the 
VAR errors. In do so, we first assume that $\gamma_{t}$ is a vector containing all the elements of $F_{t}^{-1}$ below the diagonal, stacked by rows, and include stochastic volatility, so that $\gamma_{t}$ follows:

$$
\gamma_{t}=\gamma_{t-1}+\zeta_{t}
$$

In a similar vein, $\sigma_{t}$ is a vector of diagonal elements of $D_{t}$ stacked by rows and satisfies

$$
\log \sigma_{t}=\log \sigma_{t-1}+\xi_{t}
$$

where both $\zeta_{t}$ and $\xi_{t}$ are Gaussian white noise processes with zero mean and (constant) covariance matrices $\Psi$ and $\Xi$, respectively. In order to estimate all the parameters in our model, we make a few modest assumptions as follows: a) $\Psi$ has a block diagonal structure; that is, all covariances between coefficients in different equations are zero, b) $\Xi$ is diagonal, and c) $\zeta_{t}, \xi_{t}$, and $u_{t}$ are all mutually independent. Details on the Bayesian estimation of the system are reported in the appendix.

We turn to discuss the TVP-TVAR model that is defined as follows:

$$
Y_{t}=\left[c_{1}+\theta_{1, t}(L) x_{t}+R_{1}^{1 / 2} \varepsilon_{t}\right] S_{t}+\left[c_{2}+\theta_{2, t}(L) x_{t}+R_{2}^{1 / 2} \varepsilon_{t}\right]\left(1-S_{t}\right)
$$

where $S_{t}=1 \Leftrightarrow z_{t-d} \leq z^{*}, y_{t}$ is the $n \times 1$ vector of endogenous variables, $z$ is the threshold variable that defines the state of the returns/volatility (boom/bust state), and the threshold $z^{*}$ is assumed to be the mean value of the threshold variable up to point $t-d$. The variable $d$ defines the delay with which the change in the state of the system is measured and included in the model. In this modeling setup, the propagation mechanisms between political risk and oil returns/volatility are allowed to change over time according to the state of the system. In our implementation, we consider the GPR index as the threshold variable.

The TVP-tTVAR model follows a similar definition but with a different assumption on the distribution of the innovations, and thus, we skip the discussion of the TVPtTVAR model. The estimation of both TVP-TVAR and TVP-tTVAR models starts by 
using the identical prior that is based on stochastic errors and the procedure described in the appendix.

\subsubsection{Conditional densities forecasts}

While point forecasts are useful in providing a specific estimate of the future evolution of a variable, coupling point forecasts with an explicit description of the uncertainty of the forecast is an evaluation of the quality of the forecast. Given the probabilistic approach of our forecasting, the entire conditional probability distribution (or predictive density as used in the literature) provides information on the likelihood of the appearance of different economic phenomena.

In order to examine the uncertainty around our point forecasts, we evaluate the respectful conditional probability density forecasts. Diebold et al. (1998) introduce the probability integral transform (PIT) into economics as a tool to test whether the empirical predictive distribution of empirical models matches the true, unobserved distribution that generates the data. More formally, with a given information set at time $t$ denoted as $\mathfrak{I}_{t}$ and $G$ be the length of the rolling window, we estimate the conditional probability function (PDF) $\left\{\hat{\varphi}_{t+h}\left(\hat{g}_{t+h} \mid \mathfrak{I}_{t}\right)\right\}_{t=G}^{n}$ in order to obtain the conditional forecast PDF. We then test whether the forecasted distribution $\widehat{\varphi}_{t+h}$ matches the actual unobserved distribution that generates the data $\hat{g}_{t+h}$ with the specification test for the conditional predictive densities proposed by Rossi and Sekhposyan (2018, RS hereafter). The RS specification can also be used to test

$H^{\prime}{ }_{0}$ : the empirical conditional predictive density is correctly specified in matching the actual data distribution.

Thus, a rejection of the null hypothesis ${H^{\prime}}_{0}$ implies that the model cannot capture the true data generating mechanism. Given the fact that the test uses the full distribution and not only uses a point forecast to provide higher power to the test in comparison to the tests developed by Diebold and Mariano (1995), Clark and West (2007), and McCracken (2007).

For each PDF $\hat{\varphi}_{t+h}$, the Probability Integral Transformation (PIT) is the Cumulative Density Function (CDF) evaluated at:

$$
k_{t+h}=\int_{-\infty}^{\hat{g}_{t+h}} \hat{\varphi}_{t+h}\left(k \mid \mathfrak{H}_{t}\right) d k=\hat{\varphi}_{t+h}\left(\hat{g}_{t+h} \mid S_{t}\right) .
$$


Following the CDF of the PDF given in Equation (6), we are interested in estimating the probability of the out-of-sample forecasts of length $P$ such that

$$
\begin{gathered}
\Psi_{P}(r)=P^{-1 / 2} \sum_{t=G}^{n} \xi_{t+h}(r), \\
\text { given }\left\{\begin{array}{c}
\xi_{t+h}(r)=\left(1\left\{\varphi_{t+h}\left(\hat{g}_{t+h} \mid \mathfrak{H}_{t-G+1}^{t}\right) \leq r\right\}-r\right) \\
r \in[0,1]
\end{array}\right.
\end{gathered}
$$

The null hypothesis could be formally stated as

$H_{0}: \hat{\varphi}_{t+h}\left(\hat{g}_{t+h} \mid \mathfrak{H}_{t-G+1}\right)=\hat{\varphi}_{0}\left(\hat{g}_{t+h} \mid F_{t}\right)$

where $F_{t}$ is the information included in the true data generating process, $\mathfrak{H}_{t-G+1}$, the information is included in the estimation model and $\hat{\varphi}_{0}\left(\hat{g}_{t+h} \mid F_{t}\right)=\operatorname{Pr}\left(\hat{g}_{t+h} \leq g \mid F_{t}\right)$ is the probability distribution of the null hypothesis $H_{0}$. We employ the following test statistics:

$$
\begin{aligned}
& K_{P}^{C S}=\sup \left(\Psi_{P}(r)^{2}\right), r \in[0,1], \\
& C_{P}^{C S}=\int_{0}^{1} \Psi_{P}(r)^{2} d r,
\end{aligned}
$$

for testing $H_{0}$. The critical value of the test statistic $K_{P}^{C S}$ is estimated asymptotically. To complement the use of critical value, the test possesses a graphical implementation. After plotting the CDF of the PITs, together with the CDF of the uniform $r$ (the $45^{\circ}$ line) and the critical value lines $r \pm \sqrt{K_{0.05}^{C S} / P}$, one can test whether the test statistic exceeds the critical value of the test.

\section{Empirical results}

The estimation of all the models being used in our paper is based on a rolling window process with length of $G=120$ observations (10 years) and a rolling window of 1 month. We examine the forecasting horizons of $1,3,6$, and 12 months ahead to measure the forecasting ability of each model in the out-of-sample forecasting. According to the $\mathrm{SIC}^{3}$, the lag order for all return models is fixed at $1 \mathrm{lag}$, while the same criterion suggests to fix the lag order at 3 lags for the volatility models. In Table

\footnotetext{
${ }^{3}$ We use the SIC criterion given that it leads to a smaller lag order in comparison to the AIC and the HQ criteria. Including less lags in our models relieves some of the intense computational cost in training our Bayesian models.
} 
2, we report the Root Mean Square Errors (RMSEs) of the point forecasts (mean values of the distribution) for oil return from the various models.

\begin{tabular}{|c|c|c|c|c|c|c|}
\hline & RW & $\begin{array}{l}\text { TVP- } \\
\text { VAR }\end{array}$ & $\begin{array}{l}\text { TVP- } \\
\text { tVAR }\end{array}$ & $\begin{array}{l}\text { TVP- } \\
\text { TAR }\end{array}$ & $\begin{array}{l}\text { TVP- } \\
\text { tTAR }\end{array}$ & $\begin{array}{c}\text { TVP-MS- } \\
\text { VAR }\end{array}$ \\
\hline \multicolumn{7}{|c|}{ Panel A: 1-month forecasting horizon } \\
\hline Global simple & & 4.528 & 6.567 & 4.715 & 6.567 & 6.567 \\
\hline Global Terrorist acts & & 4.524 & 6.565 & 4.712 & 6.565 & 6.565 \\
\hline Global War acts & & 4.571 & 6.565 & 4.501 & 6.565 & 6.565 \\
\hline Global Broad & 10.383 & 4.512 & 6.568 & 4.898 & 6.568 & 6.568 \\
\hline Global Narrow & & 4.512 & 6.569 & 4.737 & 6.569 & 6.569 \\
\hline DMS & & 4.837 & 4.837 & 4.862 & 4.837 & 4.837 \\
\hline DMA & & 4.836 & 4.836 & 4.516 & 4.836 & 4.836 \\
\hline \multicolumn{7}{|c|}{ Panel B: 3-month forecasting horizon } \\
\hline Global simple & & 6.235 & 6.561 & 6.264 & 6.561 & 6.264 \\
\hline Global Terrorist acts & & 6.265 & 6.561 & 6.263 & 6.561 & 6.263 \\
\hline Global War acts & & 6.263 & 6.560 & 6.256 & 6.560 & 6.256 \\
\hline Global Broad & 12.238 & 6.220 & 6.563 & 6.247 & 6.563 & 6.247 \\
\hline Global Narrow & & 6.251 & 6.563 & 6.266 & 6.563 & 6.266 \\
\hline DMS & & 6.267 & 6.267 & 6.401 & 6.267 & 6.267 \\
\hline DMA & & 6.279 & 6.279 & 6.155 & 6.279 & 6.279 \\
\hline \multicolumn{7}{|c|}{ Panel C: 6-month forecasting horizon } \\
\hline Global simple & & 6.466 & 6.560 & 6.433 & 6.560 & 6.560 \\
\hline Global Terrorist acts & & 6.491 & 6.560 & 6.467 & 6.560 & 6.560 \\
\hline Global War acts & & 6.475 & 6.559 & 6.512 & 6.559 & 6.559 \\
\hline Global Broad & 12.666 & 6.460 & 6.560 & 6.445 & 6.560 & 6.560 \\
\hline Global Narrow & & 6.481 & 6.561 & 6.463 & 6.561 & 6.561 \\
\hline DMS & & 6.420 & 6.420 & 6.554 & 6.420 & 6.420 \\
\hline DMA & & 6.435 & 6.435 & 6.447 & 6.435 & 6.435 \\
\hline \multicolumn{7}{|c|}{ Panel D: 12-month forecasting horizon } \\
\hline Global simple & & 6.485 & 6.560 & 6.528 & 6.560 & 6.560 \\
\hline Global Terrorist acts & & 6.504 & 6.560 & 6.501 & 6.560 & 6.560 \\
\hline Global War acts & & 6.506 & 6.559 & 6.530 & 6.559 & 6.559 \\
\hline Global Broad & 11.977 & 6.512 & 6.560 & 6.496 & 6.560 & 6.560 \\
\hline Global Narrow & & 6.499 & 6.560 & 6.510 & 6.560 & 6.560 \\
\hline DMS & & 6.462 & 6.462 & 6.569 & 6.462 & 6.462 \\
\hline DMA & & 4.645 & 6.446 & 6.494 & 6.446 & 6.446 \\
\hline
\end{tabular}

Note: All values are multiplied by 100. The smallest RMSEs are reported in bold.

As we observe from Table 2, all models outperform the simple Random Walk model (the current price is the best forecast for the next period) but in more that 3-months ahead exhibit similar forecasting performance. The TVR-TAR model has the highest forecasting performance at the 1-month and the 3-month horizons. The DMA index adheres more closely than the other indices at the 3-months and 12-months ahead 
horizon, while DMS index at the 6-month horizon. Nevertheless, apart from the distinctive difference of the VAR and the TAR with the other models at the 1-month ahead forecasting horizon, the forecasting performance of all other models is similar (differences are observed after the $6^{\text {th }}$ digit after the decimal). Thus, we observe that on the 1-month ahead forecasting horizon the global war acts index is the most accurate in forecasting oil returns, while composite GPRs emanating from the emerging markets (given the strong demand for oil in their push for growth), are found to forecast oil returns relatively better at longer horizons. Having said that, differences across the models incorporating information of GPRs is not necessarily markedly different. In other words, the role of GPRs matter, but not how we econometrically model them in terms of their relationship with oil returns. ${ }^{4}$

An alternative research path would be the addition of additional variables as regressors in our models. However, note that, as indicated in the introduction of the paper, GPRs drive the state of the economy determining the demand side in the oil market and affects the financial markets and the oil price determination process. In other words, the GPR index is the source behind movements in macroeconomic and financial variables that are likely to act as predictors of the oil market. Hence, by construction, GPR encapsulates the information of several other economic and financial variables and can be thus used in a bivariate setting by ignoring possible other drivers of the oil market.

\footnotetext{
${ }^{4}$ While analysing rare disaster risks, Demirer et al., (forthcoming), as a part of robustness check, showed that GPRs can predict not only oil returns, but also its and volatility using a nonlinear framework. Given this, we used all our above models to also forecast realized volatility of oil (which is basically the sum of squared daily returns over a specific month, following Andersen and Bollerslev (1998)). However, the results (which are available upon request from the authors), showed that none of our models could beat the random walk. Thus, a simple guess in forecasting the next period's oil volatility will have higher accuracy than any of our models. The reason for this finding could be that none of our models accounts for volatility clustering (conditional volatility) that is a common approach in the literature of forecasting volatility, but could also be that we need high-frequency data (at least at the daily-level) for modeling and forecasting volatility resulting from GPRs. This is an area of research, which can be considered in the future, given that Caldara and Iacoviello has now developed daily global GPRs indices.
} 
Panel A: One month ahead forecast
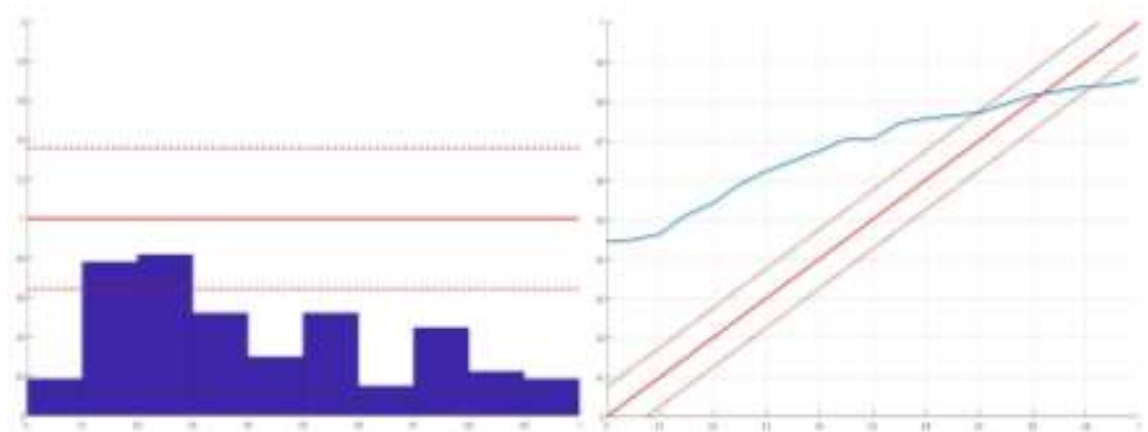

Panel B: 3 months ahead forecast
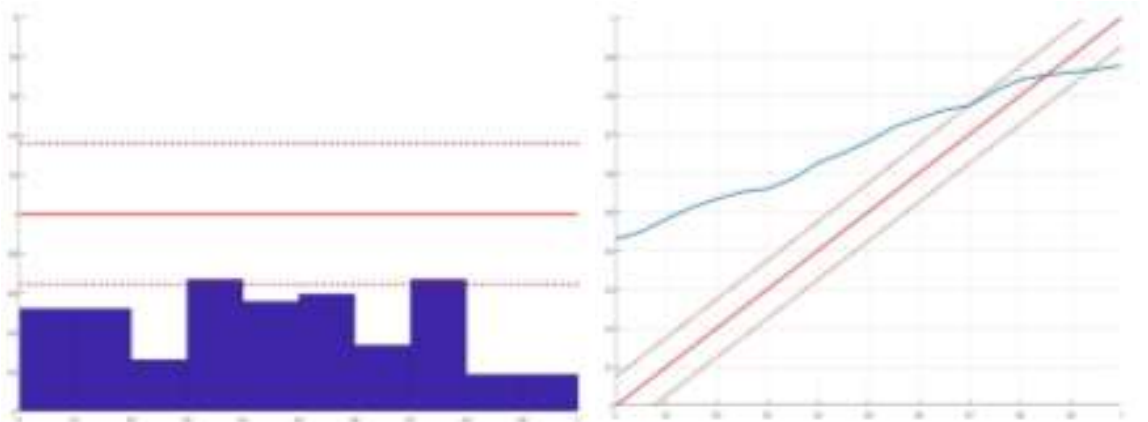

Panel C: 6 months ahead forecast
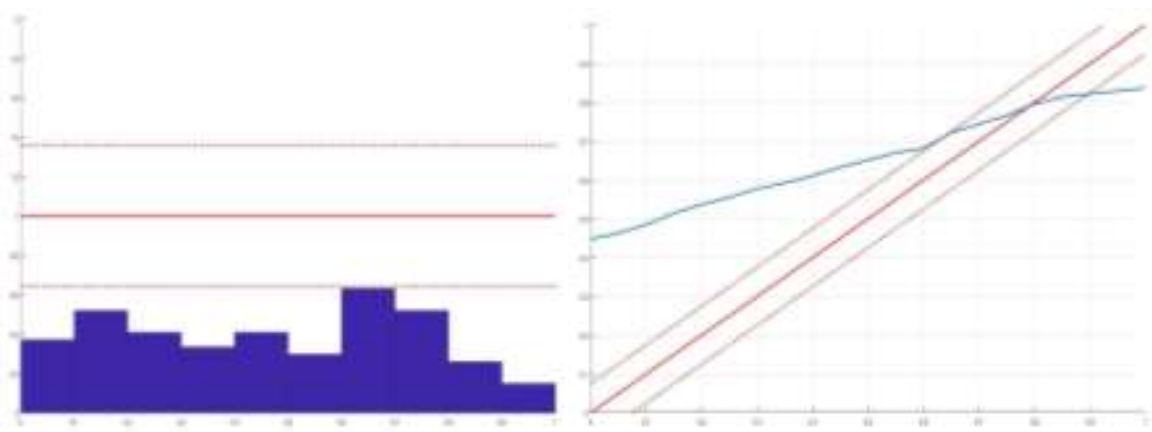

Panel D: 12 months ahead forecast

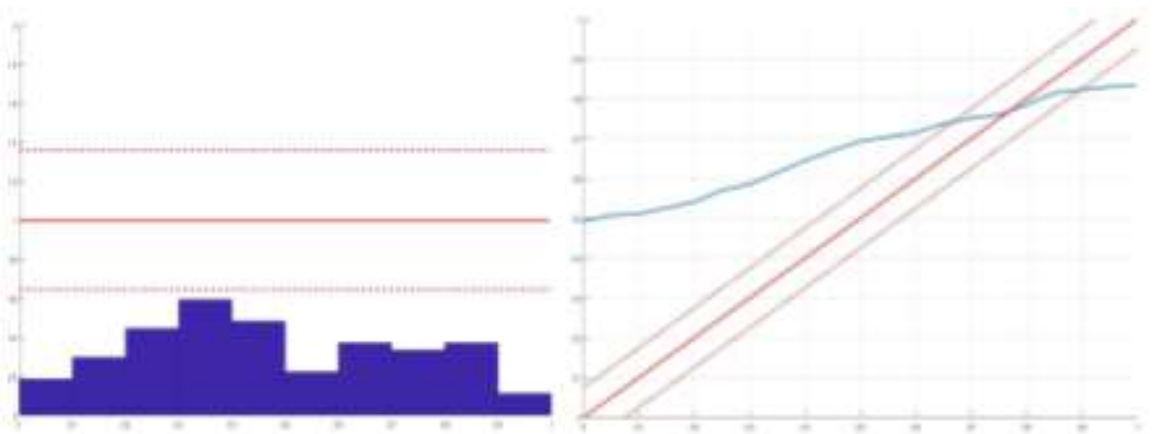

Figure 1: (left column) the figures show the pdf of the PITs (normalized) and the 95\% critical values approximated under Diebold et al.'s (1998) binomial distribution (dashed lines), constructed by using a normal approximation. (right column) The figures are the graphical representation of the RS-test. The PITs should be uniformly distributed if the null hypothesis holds true. In this situation, the CDF of the PITs should be the 45 degree line. The figure also reports the critical values of the test. If the empirical CDF of the PITs is outside the critical value lines, we conclude that the density forecast is misspecified. The GPR index used in each graph is determined by the most accurate model from Table 2. In the case of the 6-month and the 12-month ahead forecast in which we have more than one models exhibiting equal forecasting accuracy we use the TVP-VAR and the TVP-tVAR models, respectively.

Next, we move on to density forecasts obtained from the best performing point 
forecast models at each horizon for oil returns. ${ }^{5}$ In Figure 1, we depict the PITs and the RS test plots for the most accurate model per horizon, as reported via the bold figures in Table 2. In the left column we depict the pdfs of the PITs (normalized) and the $95 \%$ critical values. Panels A though D represent different forecasting horizons from of 1, 3, 6 and 12 months ahead, respectively. As we observe, most values are outside the $95 \%$ confidence intervals. In the left column we depict the graphical representation of the RS-test. In this situation, the CDF of the PITs should be the 45 degree line, while the critical values of the test are reported with the two dashed lines. Since the empirical CDFs of the PITs are outside the critical value lines, we conclude that density forecasts are mis-specified.

As we observe from Figure 1, all of our proposed models are mis-specified according to the PITs, apart from the left tail of the distribution for the 1-month ahead forecasting. In contrast, the RS test reports that in all forecasting horizons, it is the right tail of the distribution that dominates the forecasting performance of the models. Thus, relying on the more powerful RS statistic, the results tend to suggest that the models are able to foresee higher (positive) returns in the oil market but fail to foresee lower (negative) ones. Now, given that both Caldara and Iacoviello (2018), and Cunado et al. (2018) have showed that GPRs have negative impact on oil returns. Thus, the examination of the conditional densities reveal that increases in GPRs from their initial lower levels, which would imply higher conditional oil returns initially, can predict the resulting increases in oil returns thereafter more accurately compared to the lower end of the conditional distribution, which in turn, corresponds to higher initial levels of GPRs. This result reveals the fact that possibly, when GPRs are already high (and returns low), any further increase is more likely to be less informative in predicting the declines of already low oil returns. Clearly then, changes in GPRs from its high existing levels are less valuable in predicting oil returns, as the information contained in these risks does not provide clear enough information signals to be incorporated into the decision making of agents driving the oil pricing behavior.

\footnotetext{
${ }^{5}$ Results on the PIT and RS statistics for all the models are available from the authors upon request.
} 


\section{Concluding Remarks}

In this paper, we study the dynamic relationship between geopolitical risks (GPRs) of global and emerging markets and oil prices, in terms of forecasting ability of the former for the latter, based on various linear and nonlinear models for the period January 1985 to June 2017. The estimation of the models is done in a probabilistic framework, providing us with conditional density forecasts that include more information than the typical point forecasts used in the literature. Our empirical findings show that at the shortest possible horizon, i.e., 1-month ahead, global GPRs associated with wars is the most accurate in forecasting oil returns, while composite GPRs emanating from the emerging markets, forecasts oil returns relatively better at medium- to longer-horizons of 3-, 6-, and 12-months-ahead. However, differences across the linear and nonlinear models incorporating information of GPRs are not necessarily markedly different. In other words, the role of GPRs matter, but how we econometrically model them, does not seem to play a major role. Given that the literature suggests a negative relationship between GPRs and oil returns, density forecasts add another layer of information. In this regard, we find that increases in GPRs from their initial lower levels, which would imply higher conditional oil returns initially, can predict the resulting increases in oil returns thereafter more accurately compared to the lower end of the conditional distribution, which in turn, corresponds to higher initial levels of GPRs.

These results have important implications for academics, investors, and policymakers. From an academic point of view, predictability of oil returns at the upper end of its conditional distribution emanating from GPRs is an indication that the semi-strong efficiency with respect to geopolitical tensions is rejected when returns are (conditionally) high or positive. Given this, investors could use the information of GPRs to design better trading strategies to make higher profits, especially in bull rather than bear markets. ${ }^{6}$ Finally, given that, increases in GPRs, starting from initial low-levels, is likely to reduce returns in the oil market, if it was already high, would suggest a slowdown in economies that rely on oil exports, and hence, would require policymakers to take appropriate expansionary actions. At the

\footnotetext{
${ }^{6}$ Detailed discussion of market efficiency and profitability in oil markets can be found in the recent works of Lean et al., (2010, 2015).
} 
same time, for oil importers, declining oil prices (in the wake of increased GPRs) are likely to reduce domestic inflation, and just like the oil-exporters, policy makers in these economies could implement expansionary policies to boost the domestic economy, without the fear of creating higher inflation.

As part of future research, given that now daily data on GPRs have been made available by Caldara and Iacoviello (2018), it would be interesting to analyze the higher-frequency impact of these risks on oil market movements, especially volatility - a feature more likely to be observed at daily (or higher) frequencies.

\section{References}

Antonakakis, N., Chatziantoniou, I. and Filis, G., 2017a. Oil shocks and stock markets: Dynamic connectedness under the prism of recent geopolitical and economic unrest. International Review of Financial Analysis, 50(C), 1-26.

Antonakakis, N., Gupta, R., Kollias, C., Papadamou, S., 2017b. Geopolitical risks and the oil-stock nexus over 1899-2016. Financial Research Letters 23, 165-173.

Baker, S. R., N. Bloom, and S. J. Davis (2016). Measuring Economic Policy Uncertainty. The Quarterly Journal of Economics, 131(4), 1593-1636.

Balcilar, M., Bonato, M., Demirer, R., and Gupta, R., 2018. Geopolitical Risks and Stock Market Dynamics of the BRICS. Economic Systems, 42(2), 295-306.

Balcilar, M., Gupta, R., and Miller, S.M. (2015). Regime switching model of US crude oil and stock market prices: 1859 to 2013. Energy Economics, 49(C), 317327.

Baumeister, C. (2014). The Art and Science of Forecasting the Real Price of Oil. Bank of Canada Review, Spring, 21-31.

Blomberg, B., Hess, G., and Jackson, H., 2009. Terrorism and the returns to oil. Economics and Politics 21, 409-432.

Caldara, D. and Iacoviello, M. (2018) Measuring Geopolitical Risk. Board of Governors of the Federal Reserve System, International Finance Discussion Paper No. 1222.

Caldara, D., Iacoviello, M., and Markiewitz, A. (2018). Country-Specific Geopolitical Risk. Mimeo

Campbell, J.Y., (2008) Viewpoint: estimating the equity premium, Canadian Journal of Economics, 41, 1-21.

Ching-Wai C., Mumtaz, H. and Pintéra, G. (2017) Forecasting with VAR models: Fat tails and stochastic volatility, International Journal of Forecasting, 33 (4), 1124-1143.

Clark T. and West K. (2007) Approximately Normal Tests for Equal Predictive Accuracy in Nested Models, Journal of Econometrics, 138, 291-311.

Cunado, J., Gupta, R., Lau, C.K.M., and Sheng, X. (2018). Time-Varying Impact of Geopolitical Risks on Oil Prices. Department of Economics, University of Pretoria, Working Paper No. 201841. 
Degiannakis S., and Filis, G. (2017a). Forecasting oil prices. MPRA Working Paper No. 79387.

Demirer, R., Gupta, R., Suleman, T., and Wohar, M.E., Forthcoming. Time-Varying Rare Disaster Risks, Oil Returns and Volatility. Energy Economics.

Diebold, F. X., T. A. Gunther, and A. S. Tay (1998), Evaluating Density Forecasts with Applications to Financial Risk Management. International Economic Review 39(4), 863-883.

Diebold, F.X. and R.S. Mariano (1995), Comparing Predictive Accuracy, Journal of Business and Economic Statistics, 13, 253-263.

Fattouh, B., 2011. Oil market dynamics in turbulent times. Report Oxford Institute for Energy Studies. Available for download from: https://www.oxfordenergy.org/wpcms/wpcontent/uploads/2011/06/OilMarketDynamicsinTurbulentTimes.pdf.

Gupta, R., Wohar, M., 2017. Forecasting oil and stock returns with a Qual VAR using over 150 years of data. Energy Economics 62, 181-186.

Gupta, R., and Yoon S-M. (2018). OPEC News and Predictability of Oil Futures Returns and Volatility: Evidence from a Nonparametric Causality-in-Quantiles Approach. North American Journal of Economics and Finance, 45, 206-214.

Hamilton, J.D., 1983. Oil and the macroeconomy since World War II. Journal of Political Economy 91(2), 228-248.

Hamilton, J.D., 2008. Oil and the macroeconomy. In Durlauf, S., Blume, L. (eds.), New Palgrave Dictionary of Economics, 2nd edition, Palgrave McMillan Ltd.

Hamilton, J.D., 2009. Causes and consequences of the oil shock of 2007-08. Brookings Papers on Economic Activity 40(1), 215-283.

Hamilton, J.D., 2013. Historical oil shocks. In Parker, R.E., Whaples, R. (eds.), Routledge Handbook of Major Events in Economic History, New York: Routledge Taylor and Francis Group, 239-265.Lean, H.H., McAleer, M., Wong, W.K., 2010. Market Efficiency of Oil Spot and Futures: A MeanVariance and Stochastic Dominance Approach, Energy Economics 32, 979986.

Lean, H.H., McAleer, M., Wong, W.K., 2015. Preferences of risk-averse and riskseeking investors for oil spot and futures before, during and after the Global Financial Crisis,_International Review of Economics and Finance 40, 204-216.

McCracken W. (2007) Asymptotics for out of sample tests of Granger causality, Journal of Econometrics, 140, 719-752.

Mensi, W., Hammoudeh, S., Yoon, S.-M., 2014. Dynamic spillovers among major energy and cereal commodity prices. Energy Economics 43, 225-243.

Nasir, M., Rizvi A and Rossi M. (2018) A Treatise on Oil Price Shocks and their Implications for the UK Financial Sector: Analysis Based on Time- Varying Structural VAR Model, The Manchester School, 86 (5), 586-621.

Primiceri G. (2005) Time Varying Structural Vector Autoregressions and Monetary Policy. The Review of Economic Studies, 72, 821-852.

Rapach, D., and Zhou, G., 2013. Forecasting Stock Returns. Handbook of Economic Forecasting, Volume 2A, Graham Elliott and Allan Timmermann (Eds.) Amsterdam: Elsevier, 328-383. 
Rossi B. and Sehkposyan T. (2018) Alternative Tests for Correct Specification of Conditional Predictive Densities, Working Paper Series, Department of Economics and Business, Universitat Pompeu Fabra, No. 1416.

Stock, J.H., and Watson, M.W., 2003. Forecasting Output and Inflation: The Role of Asset Prices. Journal of Economic Literature, XLI, 788-829. 


\section{Appendix}

Our estimation procedure draws directly from Canova and Gambetti (2010) and Chiu et al. (2017).

\section{Priors}

We let $z^{T}$ dente the sequence of $z$ 's up to time T and let $\gamma$ be the vector containing the non-zero elements of $F^{-1}$ that are different from one and are stacked in rows and $\Xi$ a vector including all the $\Xi_{i}$, the transition density becomes

$$
p\left(\theta_{t} \mid \theta_{t-1}, \Omega\right)=N\left(\theta_{t-1}, \Omega\right) \propto I\left(\theta_{t}\right) f\left(\theta_{t} \mid \theta_{t-1}, \Omega\right),
$$

where $I\left(\theta_{t}\right)$ is an indicator function selecting non-explosive draws of $\theta_{t}$ for $y_{t}$. We assume the hyperparameters and the initial states are independent so that the joint prior is simply the product of the marginal densities. Following Cogley and Sargent (2005), we assume $P\left(\theta_{0}\right) \propto I\left(\theta_{0}\right) N(\bar{\theta}, \bar{P}), \quad P(\Omega)=\operatorname{IW}\left(\bar{\Omega}^{-1}, T_{0}\right), P\left(\log \sigma i_{0}\right)=$ $\mathrm{N}\left(\log \bar{\sigma}_{i}, 10\right), P(\gamma)=\mathrm{N}\left(0,10000 \times I_{4}\right)$, and $P\left(\Xi_{i}\right)=\operatorname{IG}\left(\frac{0.01^{2}}{2}, \frac{1}{2}\right), \quad$ where $\quad$ both $\bar{\theta}$ and $\bar{P}$ are the OLS estimates of the VAR coefficients with their variances obtained from the initial sample, $\bar{\Omega}=\lambda \overline{\mathrm{P}}, T_{0}$ is the number of observations in the initial sample (40 observations), and $\bar{\sigma}_{i}$ is the estimate of the variance of the residual in the $t$ th equation obtained by using the initial sample. The hyperparameter $\lambda$ is set to be 0.0005 for all parameters except for the constant terms of output growth, inflation, and interest rate that are set to be 0.001 . 


\section{Posteriors}

To draw realizations from the posterior density, we use the Gibbs sampler. Under regularity conditions and after a burn-in period, each iteration is composed in the following four steps to produce draws from the joint density:

- $\quad$ Step 1: $p\left(\theta^{T} \mid y^{T}, \gamma, \sigma^{T}, \Xi, \Omega\right)$

Conditional on $\left(\theta^{T} \mid y^{T}, \gamma, \sigma^{T}, \Xi, \Omega\right)$, the unrestricted posterior of the states is normal. To draw from the conditional posterior, we employ the algorithm of Carter and Kohn (1994). The conditional mean and variance of the terminal state $\theta^{T}$ can be obtained by using the standard Kalman filter recursions while for all the other states, the following backward recursions are employed:

$\theta_{t \mid t-1}=\theta_{t \mid t}+P_{t \mid t} P_{t \mid t}^{-1}\left(\theta_{t+1}-\theta_{t \mid t}\right)$ and $P_{t \mid t-1}=P_{t \mid t}-P_{t \mid t} P_{t+1 \mid t}{ }^{-1} P_{t \mid t}$ where $p\left(\theta_{t} \mid \theta_{t+1}, y^{T}, \gamma, \sigma^{T}, \Xi, \Omega\right) \sim N\left(\theta_{t \mid t+1}, P_{t \mid t+1}\right)$.

\section{- Step 2: $p\left(\gamma \mid y^{T}, \theta^{T}, \sigma^{T}, \Xi, \Omega\right)$}

Given that $\sigma^{T}$ and $y^{T}$ are known, $\varepsilon_{t}$ is known and since $u_{t}$ is a standard Gaussian white noise, we have $D_{t}^{-1 / 2} F^{-1} \varepsilon_{t}=u_{t}$ or $D_{t}^{-1 / 2} \varepsilon_{t}=D_{t}^{-1 / 2}\left(F^{-1}-I\right) \varepsilon_{t}+u_{t}$. We rewrite the ith equation as $z_{i t}=-w_{i t} \gamma_{i}+u_{i t}$ where $z_{i t}=\varepsilon_{i t} / \sqrt{\sigma_{i t}}, w_{i t}=$ $\left[\varepsilon_{1 t} / \sqrt{\sigma_{1 t}}, \ldots, \varepsilon_{i-1, t} / \sqrt{\sigma_{i-1, t}}\right]$ and $\gamma_{i}$ is the column vector formed by setting all nonzero elements of the ith row of $F^{-1}-I$. Given the normal prior, the posterior is $\gamma_{i}=$ $N\left(F_{1, i}, V_{1, i}\right)$ where $F_{1, i}=V_{0, i}\left(V_{0, i}^{-1} \gamma_{0, i}+w_{i}^{\prime} z_{i}\right)$ and $V_{1, i}=\left(V_{0, i}^{-1}+w_{i}^{\prime} w_{i}\right)$ with $V_{0, i}$ and $\gamma_{0, i}$ be the prior variance and mean, respectively. Drawing for $i=2,3,4$, we obtain a draw for $\gamma$.

- $\quad$ Step 3: $p\left(\sigma^{T} \mid y^{T}, \theta^{T}, \gamma, \Xi, \Omega\right)$

The elements of $\sigma^{T}$ are drawn by using the univariate algorithm used by Jacquier, Polson and Rossi (2004) along the approach described in Cogley and Sargent (2005) (see Appendix B.2.5 for details).

- Step 4: $p\left(\Xi_{i} \mid y^{T}, \theta^{T}, \gamma, \sigma^{T}, \Omega\right), p\left(\Omega \mid y^{T}, \theta^{T}, \gamma, \sigma^{T}, \Xi\right)$

Conditional on $y^{T}, \theta^{T}, \gamma, \sigma^{T}$ and under conjugate priors, all the remaining hyperparameters can be sampled in a standard way from Inverted Wishart and Inverted Gamma densities (Gellman et al., 1995). We perform 20000 repetitions, discard the first 15000 draws and, for inference, we keep one for every 10 of the remaining draws to break the autocorrelation of the draws. 


\section{Appendix References}

Canova F. and Gambetti, L. (2010). Do expectations matter? The great moderation revisited. American Economic Journal: Macroeconomics, 2 (3), 183-205.

Carter, C. and Kohn, R. (1994). On Gibbs sampling for state space models. Biometrika, 81 (3), 541-553.

Cogley, T. and Sargent, T. (2005). Drifts and volatilities: monetary policies and outcomes in the post WWII US. Review of Economic Dynamics, 8 (2), 262-302.

Gelman A., Carlin J. B., Stern H. S and Rubin D. B. (1995) Bayesian Data Analysis, Chapman and Hall, London.

Jacquier, E., Polson, N. and Rossi, P. (2004). Bayesian analysis of stochastic volatility models with fat-tails and correlated errors. Journal of Econometrics, 122 (1), 185-212. 